\title{
Den Blick zurück nach vorn - Plädoyer für eine verstehende Polizeiforschung
}

Anja Mensching

\begin{abstract}
Das Verhältnis der Sozialwissenschaften zur Polizei und der Polizei zu den Sozialwissenschaften ist in der Vergangenheit aus unterschiedlichster Perspektive beschrieben worden (in letzter Zeit u.a. Bornewasser 2002, Ohlemacher 2000a, Reichertz/Schröer 2003, Schneider 2000, Weitemeier 2002). Einerseits ging es dabei um die Frage, inwieweit die Sozialwissenschaften Einzug in die Polizeiausbildung gehalten haben (»Versozialwissenschaftlichung« der Polizeiausbildung, so Ohlemacher 2000a: 3 oder »stiefmütterliches Dasein«, so Krüger 1996: 225), andererseits um die Frage, inwieweit sich die Polizei dem wissenschaftlichen Einblick öffnet und es zulässt, dass Forscher an polizeilichen Einsätzen oder Vernehmungen teilhaben bzw. Akteneinsicht nehmen und Polizeibeamte/-innen zu ihrem Einsatzhandeln befragen. Mittlerweile rücken zudem Binnenverhältnisse innerhalb der Polizei vor dem Hintergrund organisationsinterner Zusammenhänge in den Blick sozialwissenschaftlichen Forschens und eröffnen so der Polizeiforschung neue Perspektiven.
\end{abstract}

\section{Dualität von Forschung sfür< und Forschung süber< die Polizei?}

W arum nur, so könnte man fragen, hat es die Polizei schwer mit den Sozialwissenschaften und jene es mit ihr? Sicherlich ist es richtig, dass die Polizei als Institution von den Sozialwissenschaften oftmals einfaches Rezeptwissen erwartete, Handlungsanweisungen, die sich 1:1 auf ihre Alltagspraxis übertragen ließen. Auch Befürchtungen, Wissenschaftler könnten durch ihre Veröffentlichungen zu viel Wissen über die Ermittlungspraxis an Gesetzesübertreter weitergeben und damit die künftige polizeiliche Tätigkeit erschweren (Reichertz 2003: 415), liegen nahe. Demgegenüber wurden Sozialwissenschaftler/-innen nicht müde zu betonen, dass sie als externe Beobachter der Polizei zunächst einmal irritierende Beschreibungen liefern, die bestenfalls eine zur Reflexion anregende andere Perspektive einführen und die polizeiinterne Gewissheiten notwendigerweise verstören. Durch den Rückzug auf wissenschaftliche Interessen verweigerte man sich allzu oft einer praxisbezogenen Vermittlung von Forschungsergebnissen. Dem Wunsch nach Eindeutigkeit seitens der Polizei konnte - so scheint es - von sozialwissen- schaftlicher Seite nur der Rückzug auf Mehrdeutigkeit entgegengesetzt werden. Dies allein für das spannungsreiche Verhältnis zwischen Sozialwissenschaften und Polizei verantwortlich zu machen, wäre jedoch zu einfach. Vielmehr beruht das problematische Verhältnis - so die diesen Artikel leitende Annahme - auch auf Missverständnissen dessen, was sozialwissenschaftliches Verstehen meint und leisten kann und damit auf inkompatiblen Erwartungen der Polizei an die Sozialwissenschaften und umgekehrt, aber auch auf den Erwartungen von Sozialwissenschaftlern/-innen an sich selbst.

Insbesondere wenn man die frühe Kritische Kriminologie betrachtet, wird eines dieser Missverständnisse offensichtlich, das auf einer durch Verständnis (statt Verstehen) geprägten Forschungsperspektive beruhte: Während man sich gegenüber devianten Subkulturen und abweichenden Karrieristen bzw. gegenüber als solchen Bezeichneten moralischer Wertungen enthielt und damit die $\mathrm{Zu}$ schreibungen staatlicher Kontrollinstanzen ablehnte (z.B. Becker 1970, Feest/Blankenburg 1972, Brusten/Malinowski 1975), galt diese Perspektivenübernahme nicht für die Analyse des Handelns der Kontrollinstanzen. Auch Meuser und Löschper formulie- ren dies rückblickend so: »In dem Maße, wie die Etikettierten reingewaschen werden, geraten umgekehrt die Vertreter der Kontrollinstanzen gleichsam auf die Anklagebank. Das geschieht zum Teil explizit, viel öfter aber - und das ist in methodologischer Hinsicht bedeutsamer - implizit dadurch, dass die Maxime des verstehenden Nachvollzuges von Handlungsmotivationen und -relevanzen hier nicht mit der gleichen Stringenz die Forschung bestimmt, wie das rauf der anderen Seite< der Fall ist." (Meuser/Löschper 2002: Absatz 12)

\section{Verstehende Polizeiforschung}

Empirische Forschung - im Sinne einer verstehenden Perspektive - erfordert weder Verständnis noch Parteinahme, weder moralische Standortbestimmung noch Sprachrohrfunktion, sondern methodisch kontrolliertes Fremdverstehen. Die Handlungslogiken des untersuchten Feldes sollen nachvollzogen werden und die Sinnhorizonte der Untersuchten rekonstruiert, nicht aber beoder entwertend eingeordnet werden. Verwechselt man Verständnis mit Verstehen, so wird eine unvoreingenommene Analyse polizeilichen Handelns unmöglich. Es geht nicht um die Frage: »whose side are we on?« (Becker 1970), sondern um empirische Argumente. Die vermeintlich unumgängliche Dualität von Forschung für die Polizei und über die Polizei, die derzeit viel diskutiert wird (u.a. Ohlemacher/Liebl 2000b, Ohlemacher 2003: 140ff., Reichertz 2002: Absätze 64ff. und Reichertz 2003: 422ff.), ist überwindbar. Man kann in der Polizei über die Polizei forschen. Forschung für die Polizei muss nicht Forschen wider (besseren) kriminologischen Wissens sein. Anschlussfähigkeit an polizeiinterne Diskurse und >polizeitaugliche Ergebnisdarstellung ist auch das Ziel der Forschung über die Polizei. Denn die angebliche Dualität ist in der wissenschaftlichen Alltagspraxis eine bunte Vielfalt verschiedenster Forschungsprogramme mit differierenden Ansprüchen, Adressaten und Zielsetzungen.

Um dies zu verdeutlichen, soll nachfolgend aus einer Perspektive des verständnisabstinenten Verstehens ein Zugang über qualitativ-rekonstruktive Forschungsmethoden entworfen werden (vgl. Bohnsack 2003; Bohnsack/Nentwig-Gesemann/ Nohl 2001; Reichertz/Schröer 2003), der an den Handlungs- und Orientierungsmustern der Beforschten ansetzt. Dem liegt die Annahme zugrunde, dass die veränderte Perspektive eine Erweiterung der Themen und Fragestellungen innerhalb der empirischen Polizeiforschung zur Folge haben wird - es tritt sozusagen zum Außen (der Analyse der Verhältnisse zwischen Polizei und Bürgern, Medien, Politik) verstärkt ein Innen (die Analyse der Binnenverhältnisse innerhalb der Organisation Polizei zwischen Arbeitsbereichen, Hierarchieebenen, Dienststellen). Bisher unterbelichtete Themen wie z.B. Mikroanalysen zu polizeilichen Hierarchiebeziehungen, zum Kommunikationsfluss auf den Dienststellen oder zum Verhältnis zwischen jüngeren und älteren Polizeibeamten/-innen innerhalb der Polizeiforschung sollen mehr Aufmerksamkeit erlangen. Zusätzlich zu der in der bundesdeutschen Polizeiforschung gängigen Analyse von Interaktionen mit Instanzen sozialer Kontrolle (z.B. zwischen Bürgern und Polizisten/-innen - u.a. Behr 1993, Dreher/Feltes 1996, Hüttermann 2000, Krasmann 1993, Steffen/Polz 1991, Walter/Wagner 1997, Wolke/Walter 2000) wäre eine in- 
tensivere wissenschaftliche Beschäftigung mit Handlungen und Entscheidungen, also Interaktionen in derartigen Institutionen zwischen Organisationsangehörigen (z.B. zwischen Polizisten/-innen) wünschenswert. Die Analyse polizeilicher Handlungspraktiken im Umgang mit dem Bürger, den Medien bzw. Erwartungen der Öffentlichkeit allgemein sollte um Forschungen ergänzt werden, die sich mit den diese Praktiken hervorbringenden und festigenden organisationsinternen Handlungslogiken und -kulturen befassen.

Eine um die dezidierte Analyse des Polizeiinneren erweiterte empirische Polizeiforschung kann die aktuelle Entwicklung einer intensiveren Selbstbeobachtung der Polizei aufgreifen, bestärken und beobachten. So beschreiben es auch polizeiinterne Sozialwissenschaftler: "Die Polizei macht sich zunehmend selber zum Gegenstand der Betrachtung und Forschung. Neben den vielfältigen Konfliktfeldern im Umgang mit dem Bürger erleben wir als in der Lehre und Forschung tätige Sozialwissenschaftler eine immer stärkere Beschäftigung mit polizeiinternen Problemen.« (Buchmann 1995: 140)

Unter diesem Vorzeichen wird eine intensivere Auseinandersetzung der empirischen Polizeiforschung mit Organisationskultur(en) in der Polizei nötig, die das Handeln von Polizeibeamten/-innen prägen. Dieser Zugang basiert auf der Vorstellung, dass polizeiinterne Organisationskulturen das Handeln von Polizeibeamten/-innen in stärkerem Maß beeinflussen als formale Vorgaben der Organisationsstruktur (etwa Dienstgrade, Funktionen, Kommunikation auf dem Dienstweg etc.).

Eine Organisation - wie die Polizei - wird dann als kulturelles Gebilde begriffen und somit der Kulturbegriff zur Basismetapher der Interpretation bzw. zur "Lese- und Interpretationshilfe « (Hervorhebung im Original; Bardmann 1994: 344). Dabei geht es nicht um die Vorstellungen in den Köpfen der Organisationsmitglieder, nicht um individuelle Ansichten, sondern um die sich in Geschichten, Anekdoten, Legenden, Metaphern etc. verdeutlichenden gemeinsamen Orientierungen bestimmter Gruppen innerhalb der Organisation Polizei.
Der Blick auf Organisationskultur(en) rückt Prozesse der Sinnkonstruktion in Organisationen auf der Ideen-, aber insbesondere auf der Interaktionsebene in den Mittelpunkt. »Mit einem solchen Begriff der Organisationskultur wird die Aufmerksamkeit auf die formellen und informellen, die sichtbaren und unsichtbaren, die bewußten und unbewußten, die rationalen und affektiven, die geplanten und spontanen, die gewollten und die nichtintendierten Elemente einer Organisation gerichtet, die direkt oder indirekt das Verhalten beeinflussen und für das tatsächliche alltägliche Tun Bedeutung haben.« (Bardmann ebenda) Die Kulturmetapher lenkt zum einen die Aufmerksamkeit auf die symbolische Bedeutung von Aspekten des Organisationsablaufs und identifiziert zum anderen gemeinsame Bedeutungssysteme, auf denen Gruppenbildung in Organisationen beruht (vgl. Morgan 1997: 191ff.).

Ein verstehender Zugang zu differierenden kulturellen Orientierungen innerhalb der Polizei kann dabei an der von Behr ausgearbeiteten Differenz einer nach außen kommunizierbaren Polizeikultur der ’kopfarbeitenden Polizisten $<$ und einer handlungsleitenden Polizistenkultur der shandarbeitenden Polizisten (vgl. Behr 2000) anknüpfen, um diese weiterzuentwickeln in Hinblick auf unterschiedliche polizeiliche Handlungsfelder oder interne Hierarchiezugehörigkeiten. Ebendies versucht der qualitative Teil des vom Kriminologischen Forschungsinstitut Niedersachsen durchgeführten Projektes mit dem Titel »Polizei im Wandel« (Leitung: Prof. Dr. Thomas Ohlemacher), der sich über das Gruppendiskussionsverfahren und ergänzende themenzentrierte Interviews mit Polizeibeamten/-innen den Binnenverhältnissen oder Organisationskulturen innerhalb der niedersächsischen Polizei, insbesondere der Kommunikation zwischen Angehörigen verschiedener hierarchischer Ebenen, zuwendet. Da hier nicht der Raum ist, das methodische Vorgehen im Einzelnen oder die bisher veröffentlichten Ergebnisse darzustellen, sei an dieser Stelle darauf hingewiesen, dass das qualitative Teilprojekt versucht, einen verstehenden Zugang zu differierenden Organisationskulturen in der Polizei zu erschließen, der mit einer konsequent organisationssoziologischen Perspektive arbeitet, d.h. nicht die Denkmuster einzelner Polizisten/innen, sondern kollektiv geteilte Orientierungen verschiedener Gruppen innerhalb der Polizei zu identifizieren sucht und in einem zweiten Schritt danach fragt, wie diese unterschiedlichen Vorstellungen innerhalb der Polizei integriert werden.

So konnten z.B. die ersten Analysen im qualitativen Teil des erwähnten Projektes die in den Gruppendiskussionen geäußerte Kritik der Polizisten/-innen am Bedarfsorientierten Schichtdienstmanagement (BSM) konkretisieren. Diese neue Form der Schichtdienstorganisation, die im Zuge der niedersächsischen Polizeireform der 1990er Jahre eingeführt wurde und die festen Schichtstrukturen zugunsten einer flexiblen Diensteinteilung der einzelnen Beamten/-innen im Einsatz- und Streifendienst auflöst, wurde in den Gruppendiskussionen vor allem hinsichtlich der Frage der damit verbundenen Folgen für das, Schichtdienstgefüge' verhandelt. Die Abwehr gegenüber dem neuen Schichtmodell des BSM resultierte dabei nicht daraus, dass es als sachlich unangemessen für dieses polizeiliche Aufgabenfeld angesehen wurde - hier betonte man vielmehr die Notwendigkeit bedarfsorientierten Personaleinsatzes, sondern das BSM wurde aufgrund von Befürchtungen hinsichtlich negativer Auswirkungen auf die durch Nähe und Verlässlichkeit geprägten Kollegengruppe abgelehnt. Zurück blieb ein paradoxer Eindruck: Das bedarfsorientierte Schichtdienstmanagement (BSM) verlangt - in den Augen der Polizeibeamten/-innen des mittleren und gehobenen Dienstes - den Zusammenhalt in der Gruppe der im Einsatz- und Streifendienst tätigen Beamten/-innen in besonderem Maß, den man durch das Modell insbesondere der damit verbundenen Auflösung der festen Schichten - gerade in Gefahr sieht (zur Konzeption der qualitativen Untersuchung und ersten Ergebnissen sowie $\mathrm{zu}$ den Ergebnissen der repräsentativen Fragebogenuntersuchung zur Arbeitssituation und -zufriedenheit niedersächsischer Polizisten/-innen vgl. Ohlemacher, Bosold, Fiedler et al. 2002).

\section{Chancen einer (sich selbst) verstehenden Polizeiforschung}

Eine verstehende Polizeiforschung muss sich zunächst einmal selbst verstehen, um sich der Organisation Polizei zuwenden zu können, d.h. sie muss sich über ihre theoretischen und methodischen Grundlagen, ihre Zielsetzungen und die Adressaten ihrer Forschungsvorhaben im Klaren sein. Sie sollte sich verabschieden von Sprachrohr- oder Anwaltsfunktionen jeglicher Art, sich lossagen von einer verengenden Entscheidung zwischen einer Forschung für und über die Polizei und konzentrieren auf die Analyse der Erfahrungsräume der Polizisten/-innen selbst. Erst ein Verstehen der Funktionsweise der Organisation Polizei, ihrer Binnenverhältnisse, der Wirkung und Deutung interner hierarchischer Strukturen - und hier scheint noch erheblicher Forschungsbedarf zu bestehen - kann die Außenverhältnisse der Polizei nachvollziehbar rekonstruieren. Kurz: das Außen ist nicht ohne das Innen denkbar.

Nur so werden beispielsweise Diskrepanzen zwischen einem gegenüber dem Bürger erwarteten souveränen Auftreten der Polizeibeamten/-innen, ihrer tagtäglichen Inszenierung von Sicherheit bei der Bearbeitung problematischer Alltagssituationen (wie z.B. Familienstreitigkeiten) und einer Verunsicherung aufgrund der eigenen Stellung innerhalb der Organisation Polizei sichtbar, die aus als mangelhaft erlebtem Rückhalt durch Vorgesetzte, ungewissen Zielvorgaben und Intransparenz der Vorhaben vorgesetzter Ebenen erwachsen. Die »verunsicherten Sicherheitsexperten « (vgl. Fiedler 2001), die Entscheidungen ihrer Vorgesetzten als für sie nicht einschätzbare Sicherheitsrisiken erleben, müssen diese innerhalb der Organisation Polizei erfahrene Unsicherheit und die Anforderung, sich als Experten für Sicherheit im Bürger-Bürger und Bürger-Polizei-Kontakt zu verstehen, in Einklang bringen.

Vor diesem Hintergrund dürften auch Orientierungs- und Handlungsmuster der Cop Culture (vgl. Behr 2000), d.h. der Praxis der Basispolizisten/-innen, sich auf eigene Maßstäbe des polizeilichen Handelns zu besinnen, in anderem Licht erscheinen. Eine wissenschaftliche Analyse der differierenden Orientierungen innerhalb der Polizei ermöglicht, Konflikte 
zwischen unterschiedlichen vertikalen oder horizontalen Ebenen in der Organisation $\mathrm{zu}$ durchdringen und an die dahinterliegenden Sinnkonstruktionen zu gelangen. Der Ertrag dieser Perspektive liegt vor allem darin, die handlungspraktische Seite hierarchischer Beziehungen innerhalb der Polizei untersuchen zu können, d.h. nicht die formal festgelegte Funktionsweise, sondern die handlungspraktischen Vollzüge der Hierarchieverhältnisse, sozusagen die gelebten Hierarchien zu rekonstruieren - nicht Organigramme, sondern Organisationskultur(en) in den Blick zu nehmen.

Ein derartiger Forschungszugang lässt die Chancen einer qualitativen Polizeiforschung zum Tragen kommen, die u.a. in der Beachtung der Priorität der Lebenspraxis der Untersuchten vor der wissenschaftlichen Erfassung und der anzunehmenden unmittelbareren Praxisrelevanz qualitativer vor quantitativer Forschung liegen (vgl. Asmus 2002: 41ff.). Insofern ist empirische Polizeiforschung weder Ideologiekritik noch Dienstleistung, wie Reichertz (2003: 422) treffend formuliert. Somit sollte der Blick zurück nach vorn gerichtet werden - zurück, um aus den Fehlern einer ideologisch motivierten Anti-Polizeiforschung $\mathrm{zu}$ lernen; nach vorn, um auf dem Weg einer konsequenten Empirieorientierung Polizei aus der Organisationsperspektive eingehender als bisher $\mathrm{zu}$ untersuchen. Dies erfordert zugleich Verstehen und Verständnisabstinenz im geschilderten Sinn.

\section{Literatur:}

Asmus, Hans-Joachim (2002): Qualitative Polizeiforschung: Bunte Erzählung oder sachadäquate Erkenntnis? S. 41-48 In: Manfred Bornewasser (Hrsg.): Empirische Polizeiforschung III. Herbolzheim: Centaurus

Bardmann, Theodor M. (1994): Wenn aus Arbeit Abfall wird. Aufbau und Abbau organisatorischer Realitäten. Frankfurt a. M.: Suhrkamp

Becker, Howard S. (1970): Whose side are we on? In: Jack D. Douglas (Hrsg.): The Relevance of Sociology. S. 99-111. New York: Appleton-Century-Crofts

Behr, Rafael (1993): Polizei im gesellschaftlichen Umbruch. Ergeb- nisse der teilnehmenden Beobachtung bei der Schutzpolizei in Thüringen. Holzkirchen: Felix

Behr, Rafael (2000): Cop Culture. Der Alltag des Gewaltmonopols. Opladen: Leske + Budrich

Bohnsack, Ralf, Iris Nentwig-Gesemann und Arnd-Michael Nohl (2001): Die dokumentarische Methode und ihre Forschungspraxis. Grundlagen qualitativer Sozialforschung. Opladen: Leske + Budrich

Bohnsack, Ralf (2003): Rekonstruktive Sozialforschung. Einführung in qualitative Methoden. Opladen: Leske + Budrich

Bornewasser, Manfred (2002): Kooperation trotz Abgrenzung der Institutionen: Über einige Schwierigkeiten, die die Zusammenarbeit von Wissenschaft und Polizei zu einem Wagnis werden lassen. S. 13-27 In: derselbe (Hrsg.): Empirische Polizeiforschung III. Herbolzheim: Centaurus

Brusten, Manfred und Peter Malinowski (1975): Die Vernehmungsmethoden der Polizei und ihre Funktion für die gesellschaftliche Verteilung des Etiketts „kriminell“. S. 57-112 In: Manfred Brusten und Jürgen Hohmeier (Hrsg.): Stigmatisierung 2. Zur Produktion gesellschaftlicher Randgruppen. Neuwied/Darmstadt: Luchterhand

Buchmann, Knud Eike (1995): Sozialwissenschaft in der Polizei - eine „Traumehe“? Die Polizei 5/1995, S. 140-144

Dreher, Gunther und Thomas Feltes (1996): Notrufe und Funkstreifenwageneinsätze bei der Polizei. Eine empirische Studie in drei Polizeidirektionen in Baden-Württemberg. Holzkirchen: Felix

Feest, Johannes und Erhard Blankenburg (1972): Die Definitionsmacht der Polizei. Strategien der Strafverfolgung und soziale Selektion. Düsseldorf: Bertelsmann

Fiedler, Anja (2001): Polizeiliches Handeln in Einsatzsituationen des privaten Konfliktes - die Definitionslast der „verunsicherten Sicherheitsexperten“. Eine qualitative Annäherung an polizeiliche Handlungsmuster. Unveröffentlichte Diplomarbeit am Aufbaustudium Kriminologie der Universität Hamburg

Hüttermann, Jörg (2000): Polizeialltag und Habitus. Eine sozialökologische Fallstudie. S. 157-183 In: Karlhans Liebl und Thomas Ohlemacher (Hrsg.): Empirische Polizeiforschung. Interdisziplinäre Perspektiven in einem sich ent- wickelnden Forschungsfeld. Herbolzheim: Centaurus

Krasmann, Susanne (1993): Kontingenz und Ordnungsmacht. Phänomenologischer Versuch über die Polizei. Münster/Hamburg: Lit

Krüger, Uta (1996): Überlegungen zur Reform der Polizeiausbildung an Fachhochschulen aus sozialwissenschaftlicher Sicht. der kriminalist 5/1996, S. 223-227

Meuser, Michael und Gabi Löschper (2002): Einleitung: Qualitative Forschung in der Kriminologie [26 Absätze]. Forum Qualitative Sozialforschung/ Forum: Qualitative Social Research [On-line Journal], 3(1). Verfügbar über:

http://www.qualitative-research.net/ fqs/fqs.htm [Datum des Zugriffs: 29.01.2002]

Morgan, Gareth (1997): Bilder der Organisation. Stuttgart: Klett-Cotta

Ohlemacher, Thomas (2000a): Die Polizei in schwierigem Gelände: Ein Plädoyer für eine veränderte Perspektive und neue empirische Projekte. In: Monatsschrift für Kriminologie und Strafrechtsreform $83 / 1$, S. 1-10

Ohlemacher, Thomas und Karlhans Liebl (2000b): Empirische Polizeiforschung: Forschung in, für und über die Polizei. S. 7-10 In: Karlhans Liebl und Thomas Ohlemacher (Hrsg.): Empirische Polizeiforschung. Interdisziplinäre Perspektiven in einem sich entwickelnden Forschungsfeld. Herbolzheim: Centaurus

Ohlemacher, Thomas, Christiane Bosold, Anja Fiedler, Oliver Lauterbach und Alexandra Zitz (unter Mitarbeit von Dirk Enzmann, Mirja Kleuker, Michaela Nack und Britta Pawlowski) (2002): Polizei im Wandel. Abschlussbericht der standardisierten Befragung der Vollzugsbeamten und -beamtinnen der niedersächsischen Polizei im Jahr 2001 sowie erste Ergebnisse der Gruppendiskussionen 2002. Forschungsbericht Nr. 87. Hannover: KFN

Ohlemacher, Thomas (2003): Diesseits von für und über? Verstehende Polizeiforschung verstehen. Kommentar zu Jo Reichertz und Norbert Schröer: „Hermeneutischwissenssoziologische Polizeiforschung". S. 139-146 In: Jo Reichertz und Norbert Schröer (Hrsg.): Hermeneutische Polizeiforschung. Opladen: Leske + Budrich

Pick, Alexander (1995): Polizeiforschung zwischen Wissenschaft und Scharlatanerie. Kriminalistik 11/1995, S. 697-704

Reichertz, Jo (2002): Prämissen einer hermeneutisch wissenssoziologischen Polizeiforschung [77 Absätze]. Forum Qualitative Sozialforschung/ Forum: Qualitative Social Research [On-line Journal], 3(1). Verfügbar über: http://www.qualitative-research. net/fqs/fqs.htm [Datum des Zugriffs: 29.01.2002]

Reichertz, Jo (2003): Empirisch-wissenssoziologische Polizeiforschung in Deutschland. S. 413-426 In: HansJürgen Lange (Hrsg.): Die Polizei der Gesellschaft. Zur Soziologie der Inneren Sicherheit. Opladen: Leske + Budrich

Reichertz, Jo und Norbert Schröer (Hrsg.) (2003): Hermeneutische Polizeiforschung. Opladen: Leske + Budrich

Schneider, Hans (2000): Anforderungsprofile für Polizeibeamtinnen und Polizeibeamte oder: Was erwarten Polizistinnen und Polizisten von der Soziologie? S. 27-34 In: Karlhans Liebl und Thomas Ohlemacher (Hrsg.). Empirische Polizeiforschung. Interdisziplinäre Perspektiven in einem sich entwickelnden Forschungsfeld. Herbolzheim: Centaurus

Steffen, Wiebke und Siegfried Polz (1991): Familienstreitigkeiten und Polizei. Befunde und Vorschläge zur polizeilichen Reaktion auf Konflikte im sozialen Nahraum. Kriminologische Forschungsgruppe der Bayerischen Polizei München: Bayerisches Landeskriminalamt

Walter, Michael und Andrea Wagner (1997): Alltägliches Krisenmanagement von Polizisten. Die Beseitigung des Öffentlichkeitsbezuges. Monatsschrift für Kriminologie und Strafrechtsreform 80, S. 44-58

Weitemeier, Ingmar (2002): Anforderungen und Erwartungen an die wissenschaftliche Forschung aus Sicht der Polizei. S. 3-12 In: Manfred Bornewasser (Hrsg.): Empirische Polizeiforschung III. Herbolzheim: Centaurus

Wolke, Angelika und Michael Walter (2000): Konfliktschlichtung durch Streifenwagenbesatzungen der Bonner und Kölner Polizei. Monatsschrift für Kriminologie und Strafrechtsreform 83, S. 91-110

Anja Mensching, Diplom-Kriminologin, Promotionsstipendiatin im Projekt "Polizei im Wandel « am Kriminologischen Forschungsinstitut Niedersachsen 